\title{
Thermocapillary rupture in falling liquid films at moderate Reynolds numbers
}

\author{
Evgeny Shatskiy, Evgeny Chinnov \\ Kutateladze Institute of Thermophysics SB RAS, 630090, 1 Lavrentyev Ave., Novosibirsk, Russia \\ Novosibirsk state University, 630090, 2 Pirogova Str., Novosibirsk, Russia
}

\begin{abstract}
An experimental study of the flow of a water film over a heated surface for $\mathrm{Re}=15-50$ was performed. The influence of the development of thermocapillary instability on the wave amplitudes, the deformation of the surface of the liquid film, and the formation of the first stable dry spot on the heater are investigated. It is shown that the interaction of waves with thermocapillary structures can lead to an increase in the critical heat flux corresponding to the rupture of the liquid film, as compared with the data known in the literature.
\end{abstract}

\section{Introduction}

Film flows are widely used in various industrial technologies and apparatuses, and understanding the processes occurring in flowing films is an important task for the implementation of such devices. An important factor affecting the intensity of heat transfer and the stability of films to rupture is the interaction of waves on the interphase surface with thermocapillary structures.

Hydrodynamic two-dimensional waves in isothermal fluid films are unstable to threedimensional perturbations. The length of the wave instability to transverse threedimensional perturbations decreases with increasing Reynolds number [1]. It is established that the transition from regular two-dimensional structures to three-dimensional flow is accompanied by a significant redistribution of liquid in the longitudinal direction $[2,3]$. Characteristic forms of three-dimensional structures developing during the transition are described.

While flowing along heated surfaces, in addition to the hydrodynamic instability leading to the development of the wave flow, there are also thermocapillary instabilities of various types associated with fluid transport along the interface due to the appearance of a surface tension gradient [4].

An investigation of the Marangoni effect in a heated liquid film with a developed wave motion is described in [5]. The film temperature fields were visualized by infrared thermography, and the film thickness was measured by confocal microscopy.

Investigations of the natural evolution of developed three-dimensional waves into thermocapillary-wave rivulets during heating of a vertically falling water film under conditions of small temperature gradients were performed in [6] with $\mathrm{Re}=10$. The deformation of horseshoe-shaped hydrodynamic waves as they pass through the heated region is recorded. It is shown that at the front of the three-dimensional wave, when moving 
along the heater, temperature inhomogeneities appeared.That led to the deformation of the liquid film and the formation of rivulets due to the action of thermocapillary forces.

An investigation of the dynamics of the rupture by means of a fiber-optic sensor was carried out in [7]. The influence of wettability of the substrate on the thermocapillary rupture of a liquid film was carried out in [8]. In [9] generalizing dependences on thermocapillary rupture of a film in a wide range of Reynolds numbers and sizes of the heater are given.

The aim of this work is an experimental study of the effect of the interaction of hydrodynamic waves with thermocapillary structures on the stability of the flow of a heated water film to rupture.

\section{Setup and experimental results}

The stand was a closed circulation circuit, including a reservoir with a pump, a working section, a filter, rotameters, pipelines and shut-off valves. The working area consisted of a working plate, on which a film former, a heat stabilizer and a heater were installed. The working liquid (water with a dye) was fed into the film former, which included a storage chamber, a dispenser and a nozzle with a calibrated flat slot. The liquid flowed down the plate and through the connecting channels under the influence of gravity, returning to the reservoir with the electric pump. As a heating element, a copper flat heat exchanger 150 $\mathrm{mm}$ wide and $100 \mathrm{~mm}$ long was used. A detailed description of the installation can be found in [10]. The critical heat flux corresponding to the rupture of the film was determined by the appearance of the first stable dry spot in the center of the heater.

A synchronous measurement of the surface temperature and a thickness distribution in the heating region at $\mathrm{Re}=15-50$ are performed. $\mathrm{Re}=\mathrm{W} / \rho v$, where $\mathrm{W}$ is the specific mass flow rate of the liquid, $\rho$ is the density of the liquid, and $v$ is the kinematic viscosity of the liquid. The method of investigation is described in detail in [10].

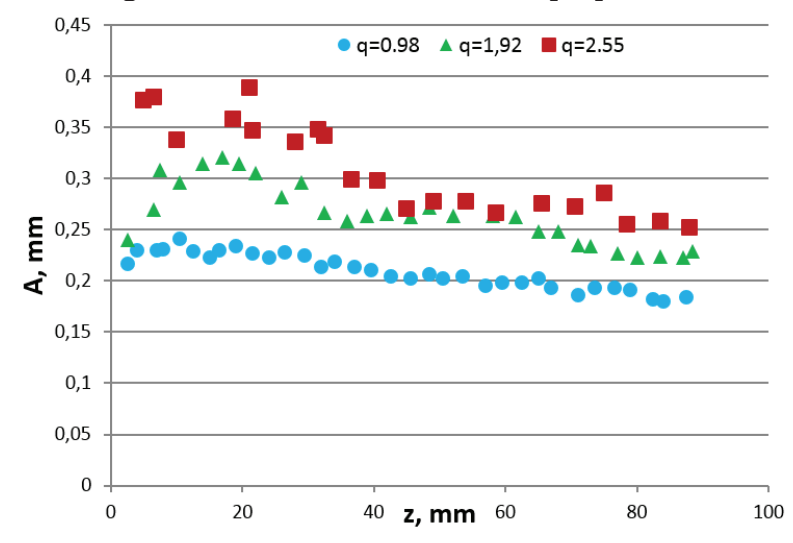

Fig. 1.Amplitude of waves in the interrivulet region for different values of the heat flux, $\operatorname{Re}=15$.

The growth of wave amplitudes was observed. Fig. 1 presents data on the amplitude of the waves in the rivulets and in the interrivulet region as a function of the film path along the heater ( $\mathrm{A}$ is the wave amplitude, $\mathrm{Z}$ is the coordinate along the heater, and $\mathrm{Z}=0$ is the beginning of the heater). It can be seen from the graphs that the amplitude of the waves in the upper part of the heater is higher than in the lower part and increases with increasing density of the heat flux.

The front of the wave, during the movement along the heater, was deformed under the action of thermocapillary forces and in the lower part of the heater the flow passed into an 
rivulet with a thin film between the rivulets. It was found for $\mathrm{Re}=15$, that for a heat flux density $\mathrm{q}>1.5 \mathrm{~W} / \mathrm{cm}^{2}$, the motion, amplitudes and deformation of the wave front differ sharply from the case $\mathrm{q}<1.5 \mathrm{~W} / \mathrm{cm}^{2}$. We see a significant change in the character of the dependence of the amplitude of the waves on the density of the heat flux, Fig. 1.

When high longitudinal gradient values are reached, which occurs at a heat flux density from 1.5 to $2 \mathrm{~W} / \mathrm{cm}^{2}$, the formation of thermocapillary structures begins. In the region of the leading edge of the heater $\mathrm{Z}<10-15 \mathrm{~mm}$, on the residual layer of liquid film, after passing the wave front, thermocapillary structures are formed in the form of a series of rivulets with a thin film between them. The distance between the structures is $\Lambda=10 \mathrm{~mm}$, which corresponds to the distance between the earlier investigated structures in regime A at other Reynolds numbers. When a new front is flowing onto such structures, a periodic inhomogeneity of the wave front along the thickness in the transverse direction appears. Such perturbation of the front leads to the formation of three-dimensional waves with a transverse distance between the ridges equal to $10 \mathrm{~mm}$. However, at $Z>23 \mathrm{~mm}$ the liquid film temperatures close to the temperature of the heater, and the temperature gradients on its surface decrease sharply. In this region three-dimensional waves merge to the lower part of the heater and the formation of thermocapillary structures in regime B appears with an average rivulet spacing of $15 \mathrm{~mm}$.

A similar picture was observed for other Reynolds numbers. So, for $\mathrm{Re}=50$, when the heat flux density was $\mathrm{q}=4 \mathrm{~W} / \mathrm{cm}^{2}$, the temperature gradients increased to $10 \mathrm{~K} / \mathrm{mm}$, which is typical for the appearance of structures of type A. Periodically, these structures were clearly visible. They were formed in the residual layer before the wave front near the leading edge of the heater. The average distance between the structures in the transverse direction corresponded to the average instability wavelength in regime $\mathrm{A}$ at lower values of Re. It was found that the thickness of the residual film layer in the region between the rivulets can be greatly reduced. Three-dimensional hydrodynamic waves were destroyed in the upper part of the heater. The determining influence on the process was provided by thermocapillary instability in the upper part of the heater.

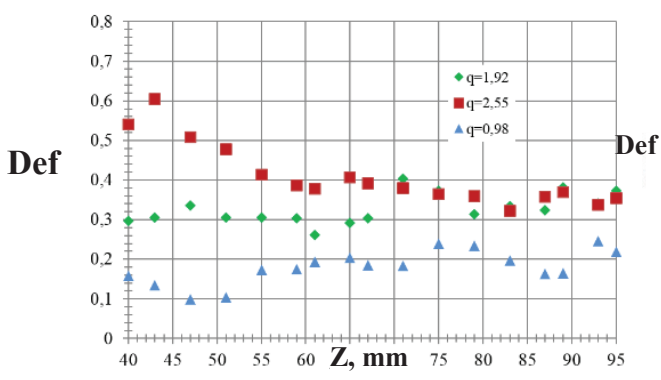

a)

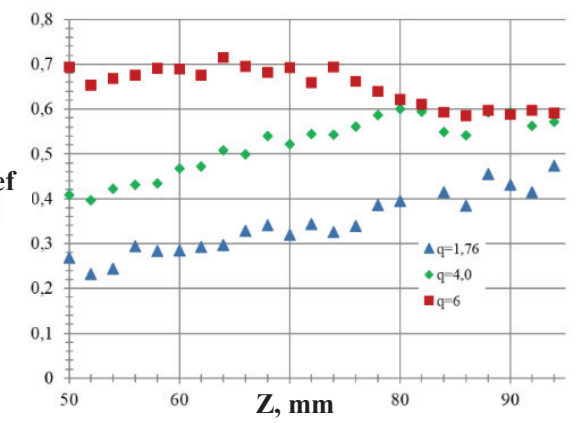

b)

Fig.2. Dependence of the film surface deformation on the longitudinal coordinate along the heater for different heat fluxes a) $\operatorname{Re}=15, \mathrm{~b}) \mathrm{Re}=50$.

In Fig. 2. The dependences of the deformation of the film surface on the longitudinal coordinate along the heater for different values of the heat flux are presented. For the quantitative description of transverse deformations in a fluid film, the criterion Def $=\left(\mathrm{h}_{\text {riv }}{ }^{-}\right.$ $\left.\mathrm{h}_{\mathrm{val}}\right) / \mathrm{h}_{0}$ is used, defined as the ratio of the difference between the average film thicknesses on the rivulet crest and in the inter rivulet region to the initial film thickness. As can be seen from the graphs, the dependence character changes at high heat flux densities. At this density the thermocapillary structures in the form of rivulets with a thin film between them form in the residual layer of liquid between the wave fronts. The interaction of waves with these structures leads to an increase in deformation at the beginning of the heater, an 
increase in the amplitude of the waves by their transformation into a rivulets and the appearance of the rivulet movement (deviation from strictly vertical flow). Further, with an increase of path of the film along the heater, the deformations decrease. At the same time, a rivulet stream with a spacing of about $15 \mathrm{~mm}$ is formed in the lower part of the heater, as well as for smaller Re.

In Fig. 3 the comparison of the density of the critical heat flux obtained in this paper with data for heaters with the boundary condition $\mathrm{q}=$ const is presented. The initial temperature of the film was maintained equal to $23{ }^{\circ} \mathrm{C}$. It can be seen that the values of the critical heat fluxes obtained in this work lie above the data for heaters, where only structures of type B are formed. The growth of wave amplitudes and deformation of the film surface after the formation of structures of type A, as well as the occurrence of the motion of rivulets in the direction across the flow leads to a substantial increase in the stability of the liquid film to rupture.

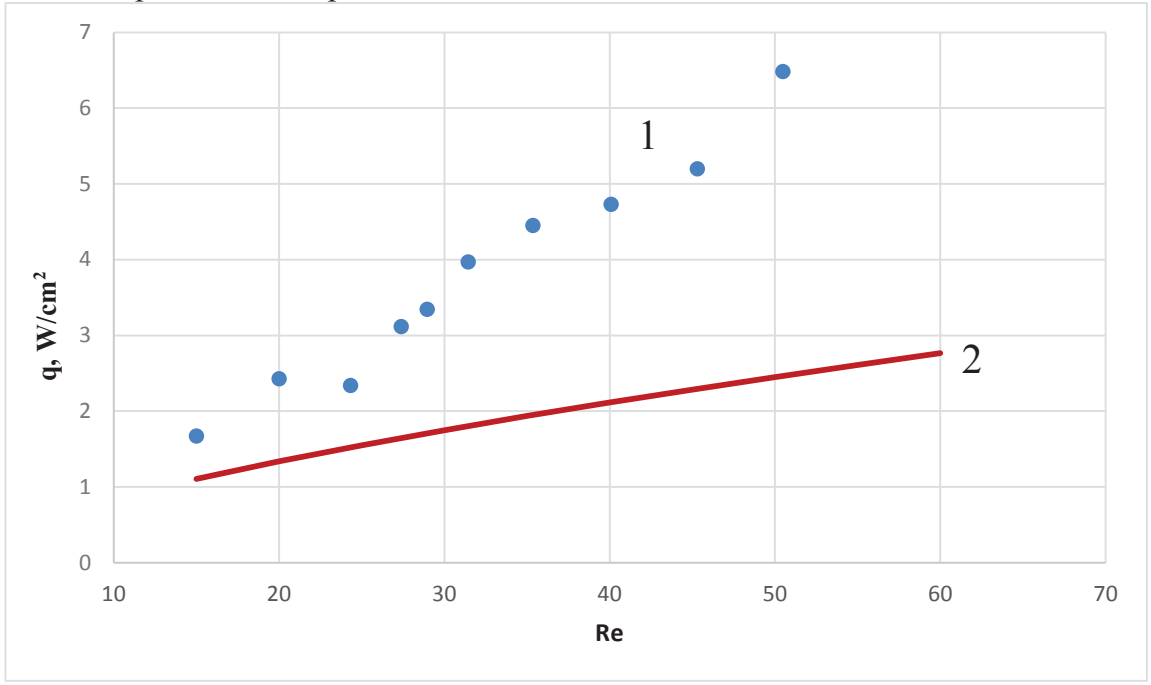

Fig.3. Dependence of the critical heat flux corresponding to the rupture of the liquid film on the Reynolds number. 1 - the critical heat flux density corresponding to the appearance of the first stable dry spot in the center of the heater, after the formation of structures of type A (the result of this work), 2 - the critical heat flux density for heaters with the boundary condition $\mathrm{q}=$ const, where only structures of the type B were formed [9].

\section{Conclusions}

Thus, it has been shown that the formation of metastable thermocapillary structures of type $\mathrm{A}$ in the upper part of the heater affects on the magnitude of the critical heat flux corresponding to rupture of the liquid film. The interaction of three-dimensional waves with thermocapillary structures leads to an increase in the amplitudes of the waves by their transformation into a jet and the appearance of the rivulet motion (deviation from strictly vertical flow). In this case, the deformation of the liquid film in the middle part of the heater increases. It is shown that the magnitude of the critical heat flux increases with the Reynolds number of the film and, at high values of Re, significantly exceeds the rupture data in the absence of thermocapillary structures of the type A. It can be concluded that for the first time a new method of influencing the film flow, leading to an increase in film stability was found. The result is important, since the discovered method of increasing the stability of liquid films can be used in industrial installations. 
The work was supported by the RF Ministry of Education and Science (Agreement No. 14.604.21.0053, project ID RFMEFI60414X0053).

\section{References}

1. C.D. Park, T. Nosoko, AIChE Journal. 49, 2715 (2003)

2. S.V. Alekseenko, V.V. Guzanov, D.M. Markovich, S.M. Kharlamov, Tech. Phys. Lett. 38, 739 (2012)

3. S.V. Alekseenko, A.V. Bobylev, V.V. Guzanov, D.M. Markovich, S.M. Kharlamov, Tech. Phys. Lett. 40, 1031 (2014)

4. Chinnov E.A., Kabov O.A., J. Appl. Mech. Tech. Ph. 44, 128 (2003)

5. V.V. Lel, A. Kellermann, G. Dietze, R. Kneer, A.N. Pavlenko, Exp. Fluids, 44, 341 (2008)

6. E.A. Chinnov, E.N. Shatskiy, Tech. Phys. Lett. 42, 997 (2016)

7. Zaitsev D. V., Rodionov D.A., Kabov O.A., Microgravity science and technology, 19, 100 (2007)

8. Zaitsev D.V., Kirichenko D.P., Kabov O.A., Technical Physics Letters, 41, 551 (2015)

9. Zaitsev, D.V., Semenov, A.A., Kabov, O.A., Thermophysics and Aeromechanics, 23, 625 (2016)

10. E.N. Shatskiy, E.A. Chinnov, High Temp., 54, 965 (2016) 